\title{
Testbeam Studies on Pick-Up in Sensors with Embedded Pitch Adapters
}

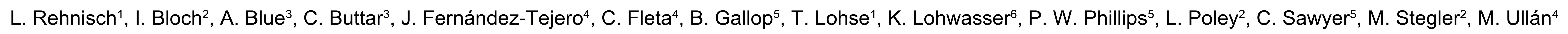

${ }^{1}$ Humboldt-Universität zu Berlin, Berlin, Germany, ${ }^{2}$ Deutsches Elektronen-Synchrotron (DESY), Zeuthen, Germany, ${ }^{3}$ University of Glasgow, Glasgow, U.K.,

${ }^{4}$ Centro Nacional de Microelectrónica (IMB-CNM), Barcelona, Spain, ${ }^{5}$ STFC Rutherford Appleton Laboratory, Didcot, U.K., ${ }^{6}$ University of Sheffield, Sheffield, U.K.

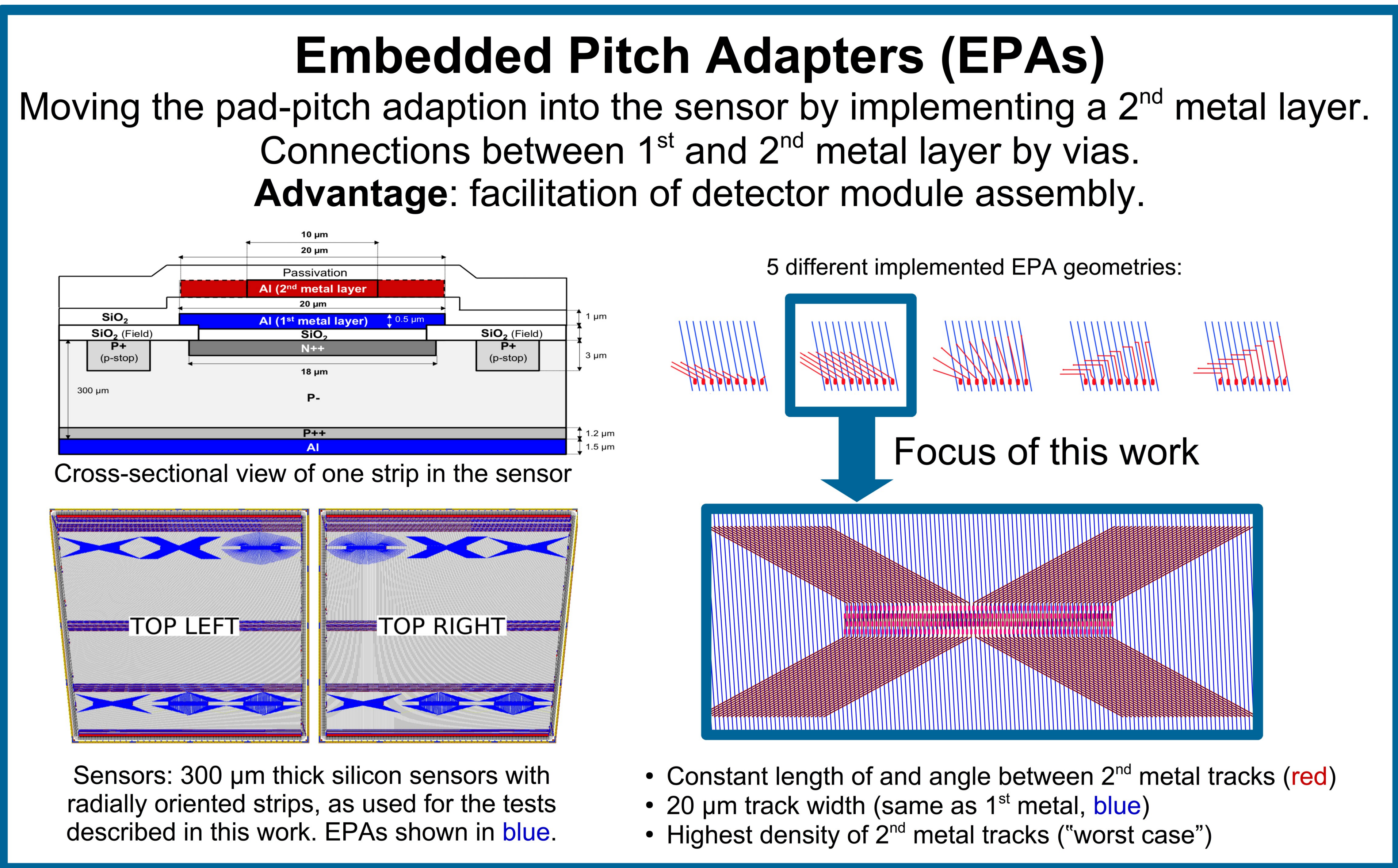

\section{Possible Challenges in Sensors with EPAs}

- Noise enhancement due to higher interstrip capacitance

- Cross-talk: coupling between tracks of the two metal layers

- Pick-up: coupling of charge induced in the silicon bulk into the EPA tracks

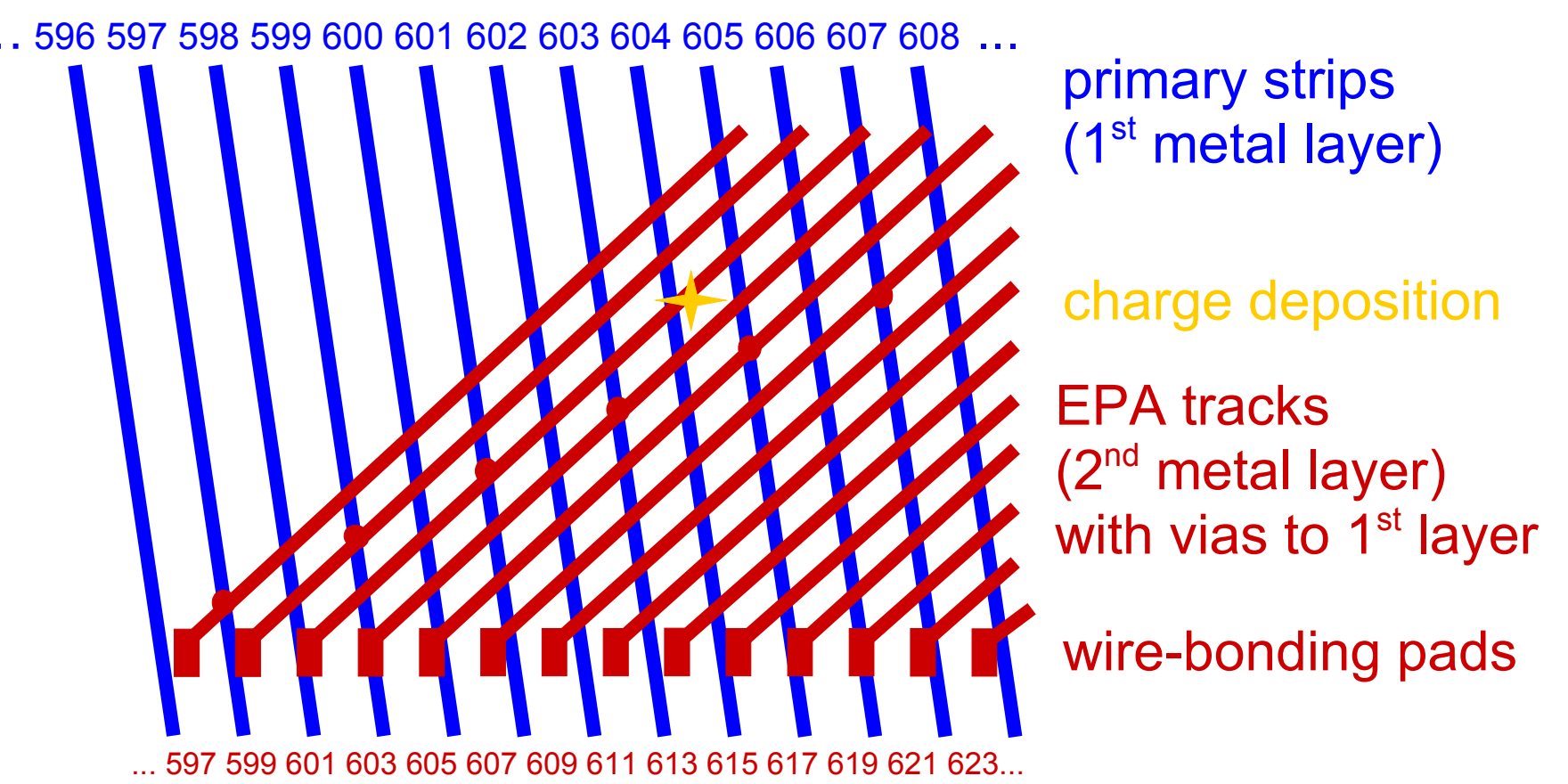

Part of the EPA geometry under study that adapts channels with odd numbers, indicating readout channel numbers of 1 st and 2

Example:

charge induced near strip 604 can be picked up by $2^{\text {nd }}$ metal track of strip 601

- Partial pick-up: signal in channel

601 and in channel 604

$\rightarrow$ fake signal

- Full pick-up: signal in channel 601

Effect restricted to area with EPA

$\rightarrow$ Investigate EPA architecture with highest density of $2^{\text {nd }}$ metal tracks

- Pick-up: Present? Threshold-dependent? Location-dependent?

\section{Experimental Setup and Performed Measurements}

Testbeam

- Synchrotron radiation from Diamond Light Source

- Monochromatic $15 \mathrm{keV}$ photon beam

- Micro-focussed beam ( $2 \mu \mathrm{m} * 3 \mu \mathrm{m}$ beam spot)

- $1.2 \pm 0.2$ photons $/ 10 \mathrm{~ns}, 51 \%$ int. probability each

\section{Implications}

- Energy deposition only $\sim 1 / 5$ that of a MIP

$\rightarrow$ no quantitative results

- Very high spatial resolution

$\rightarrow$ obtain detailed map of effects

- Avg. no. of photon interactions per event: $1.4 \pm 0.3$
Tested Device and Measurements

- ATLAS ITk strip tracker end-cap prototype module [1]

- $300 \mu \mathrm{m}$ thick high-resistivity wedge-shaped n-in-p

sensors with radially oriented strips and EPAs [2,3]

- Binary readout with ABCN-25 ASICs [4]

$\rightarrow$ no information on deposited charge

$\rightarrow$ threshold scans, hits above threshold

- Low range of discriminator thresholds probed:

$54.4 \mathrm{mV}$ to $80 \mathrm{mV}$ to measure small effects

[1] S. Kuehn et al 2017 JINST 12 P05015

[2] V. Benitez et al 2015 CERN
ATL-COM-UPGRADE-2015-027

[3] M. Ullán et al 2016 NIM A 821

[3] M. Ullán et al al 2016 NIM A 821
[4] W. Dabrowski et al IEEE Nucl. Sci. Symp. Conf.

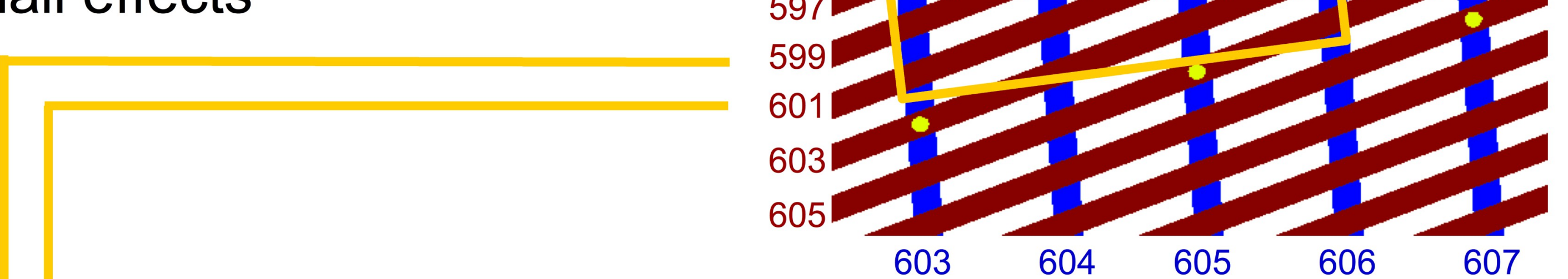

Investigated Sensor Area

- $300 \mu \mathrm{m} * 200 \mu \mathrm{m}$ in steps of $15 \mu \mathrm{m} * 10 \mu \mathrm{m}$

(primary strip pitch: $\sim 100 \mu \mathrm{m}$ )

- Covering $\sim 41^{\text {st }}$ metal and $\sim 52^{\text {nd }}$ metal tracks

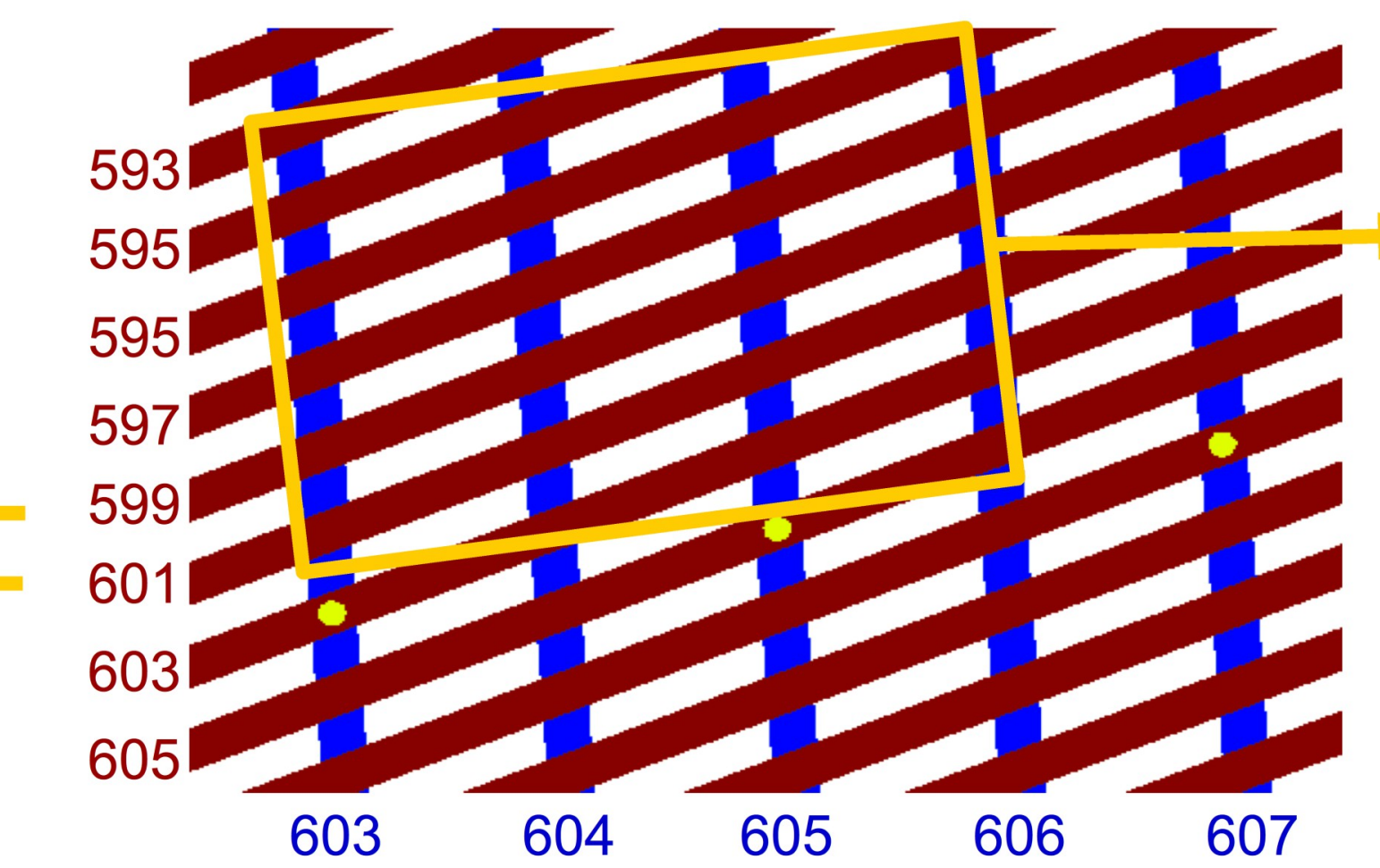

\section{Threshold/Centrality Dependence}
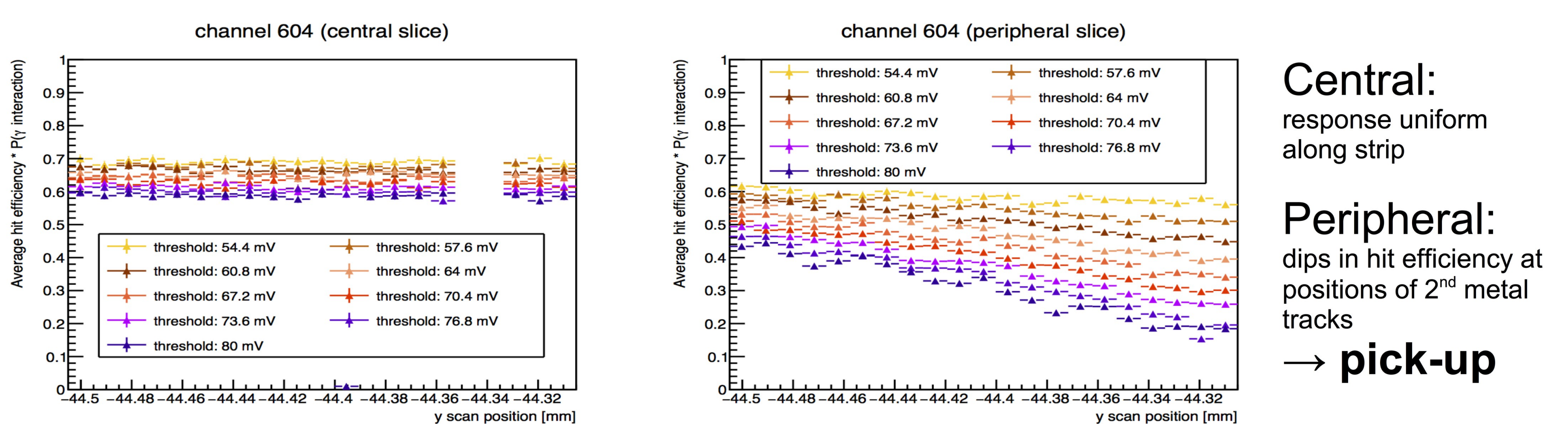

Slope along y due to slight mis-alignment
between beam movement and strip direction
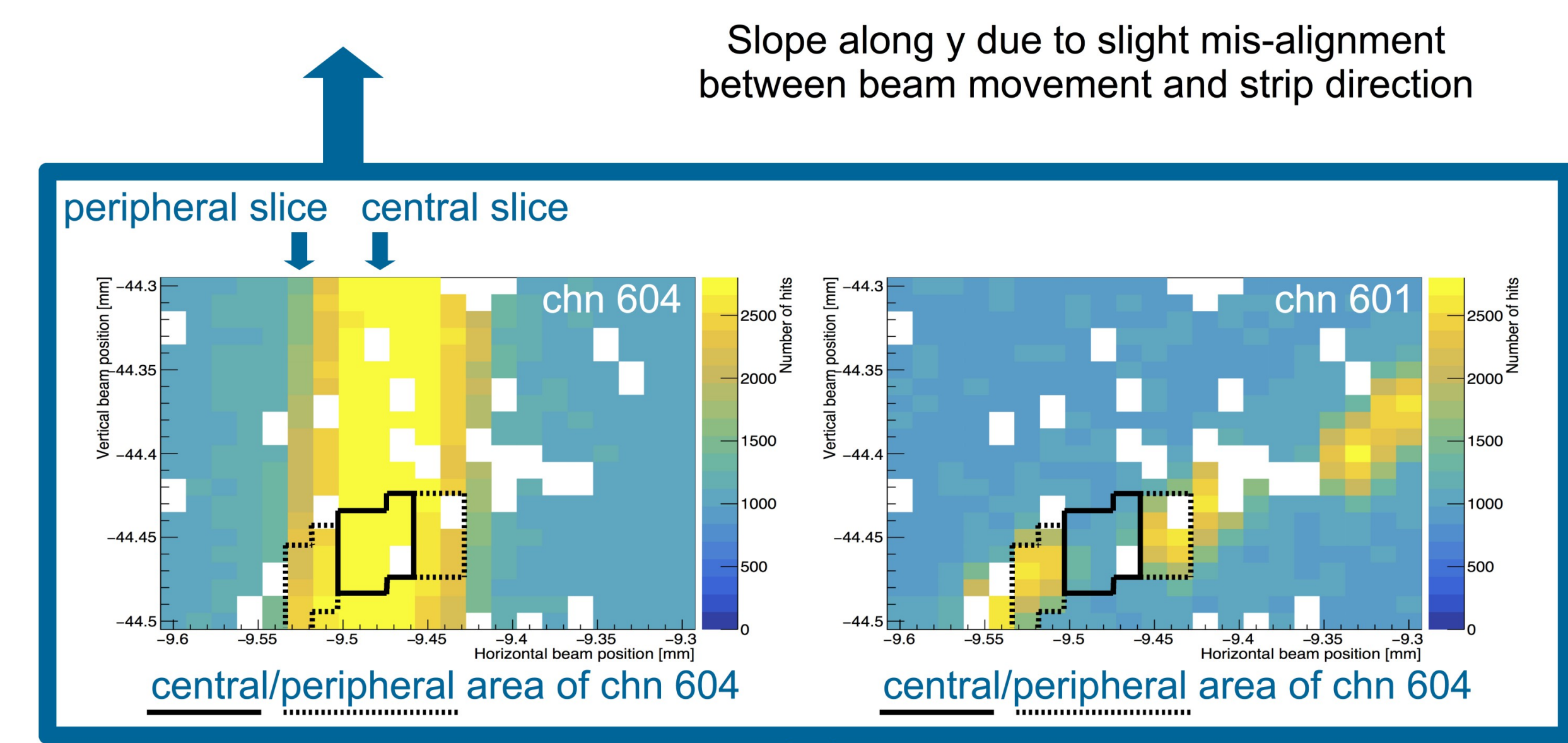

1

central area

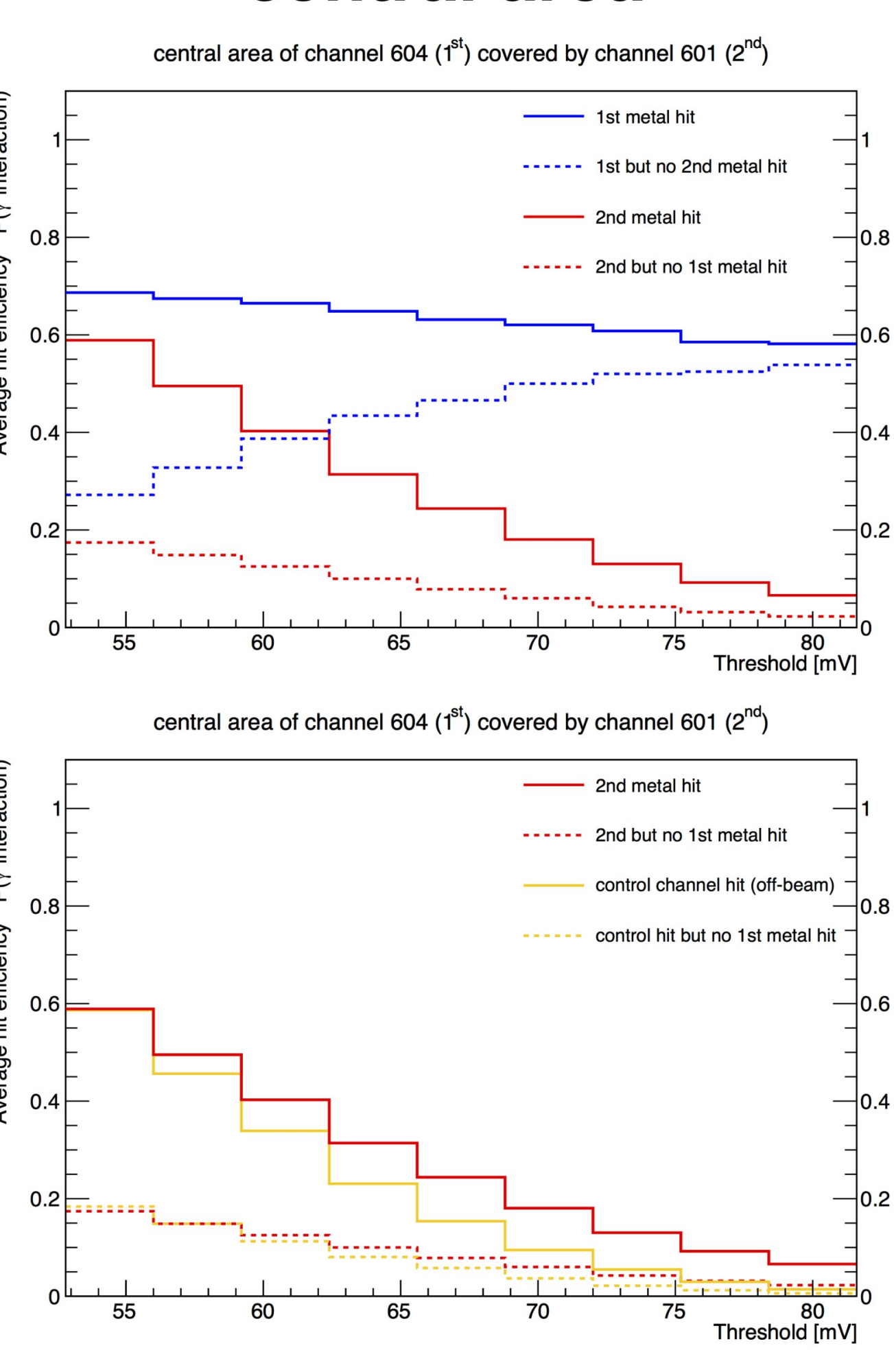

Pick-up

decreases faster with threshold

than $1^{\text {st }}$ metal hit

efficiency,

especially in

central strip area.

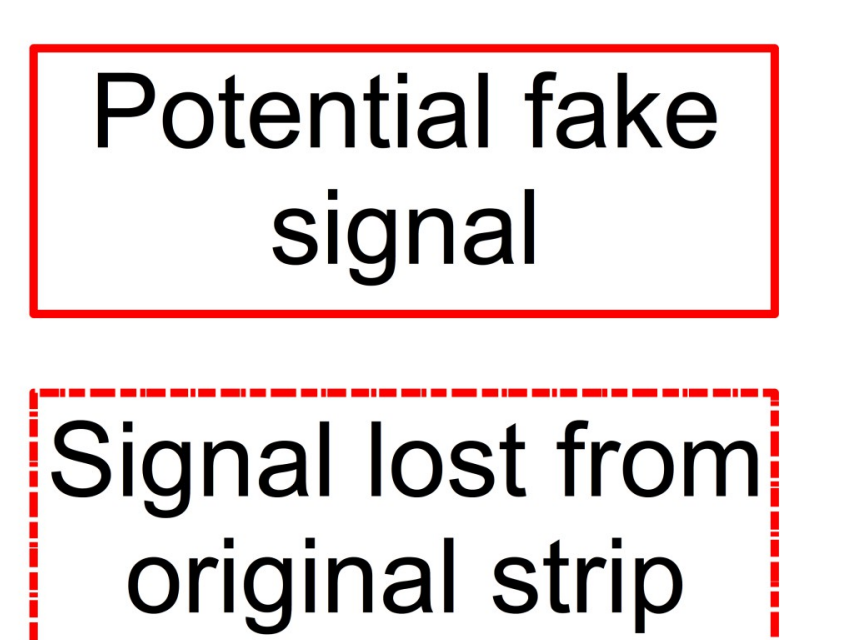

Control channel

- noise only
Visualizing the Effect

Hitmap: response of single channel over all beam positions (white areas $\rightarrow$ no data available)

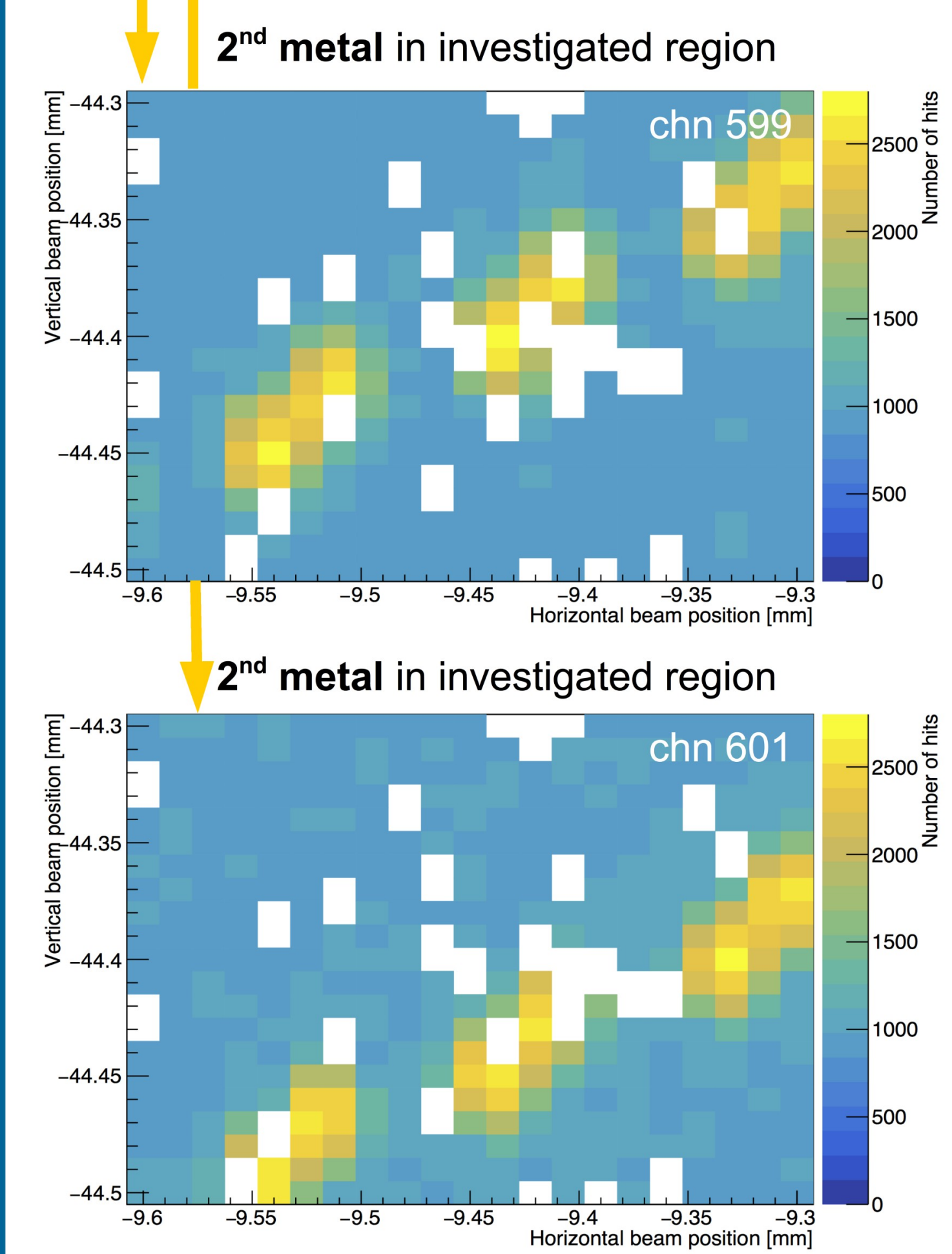

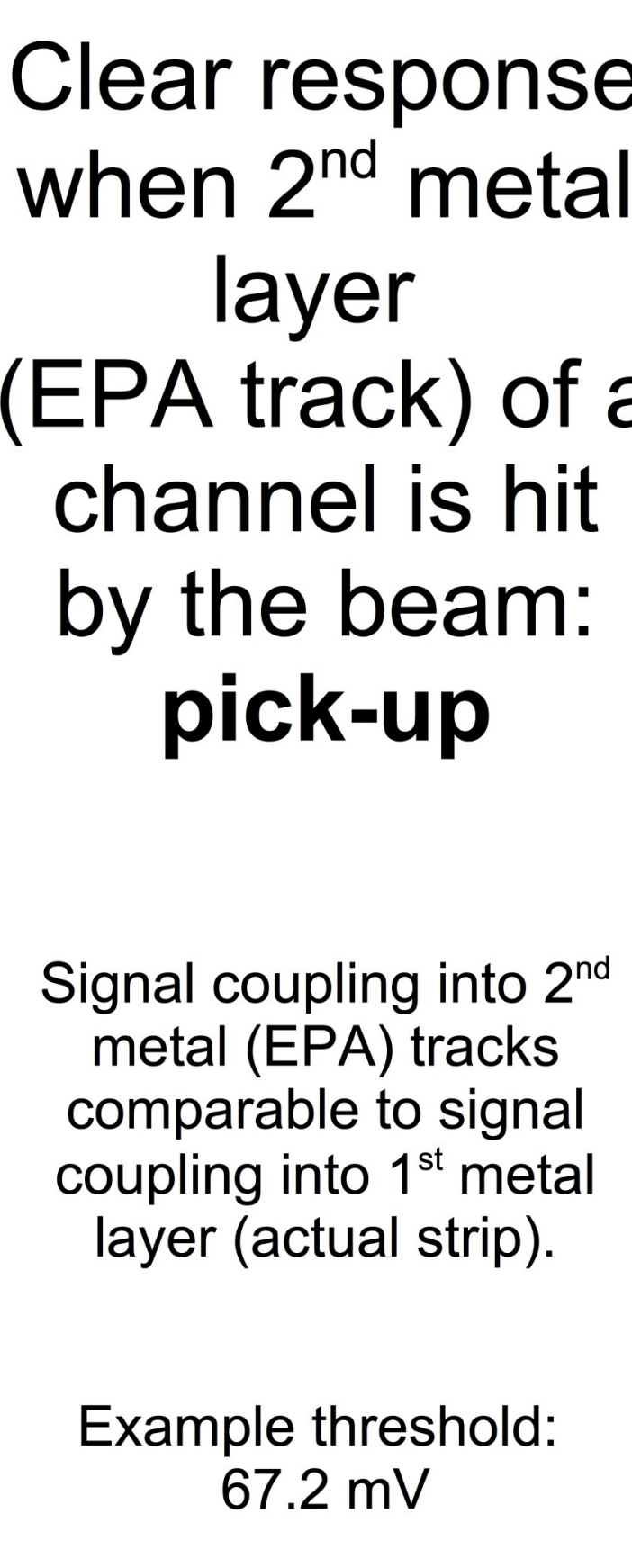

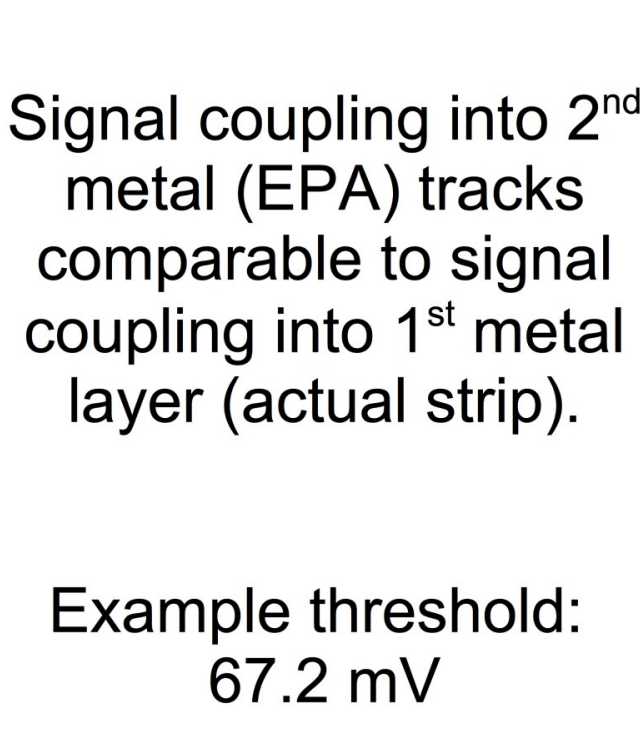

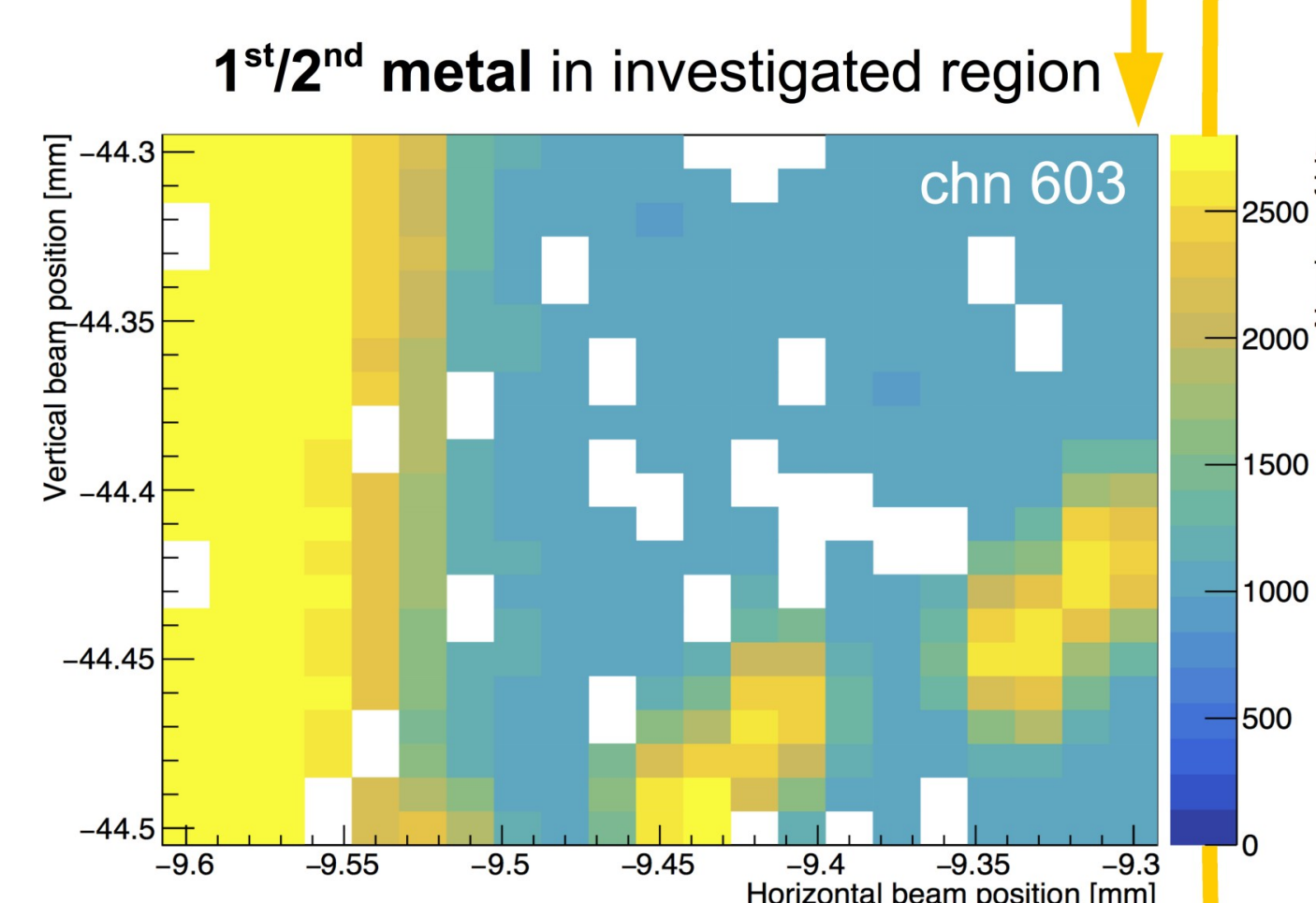

$1^{\text {st }}$ metal in investigated region

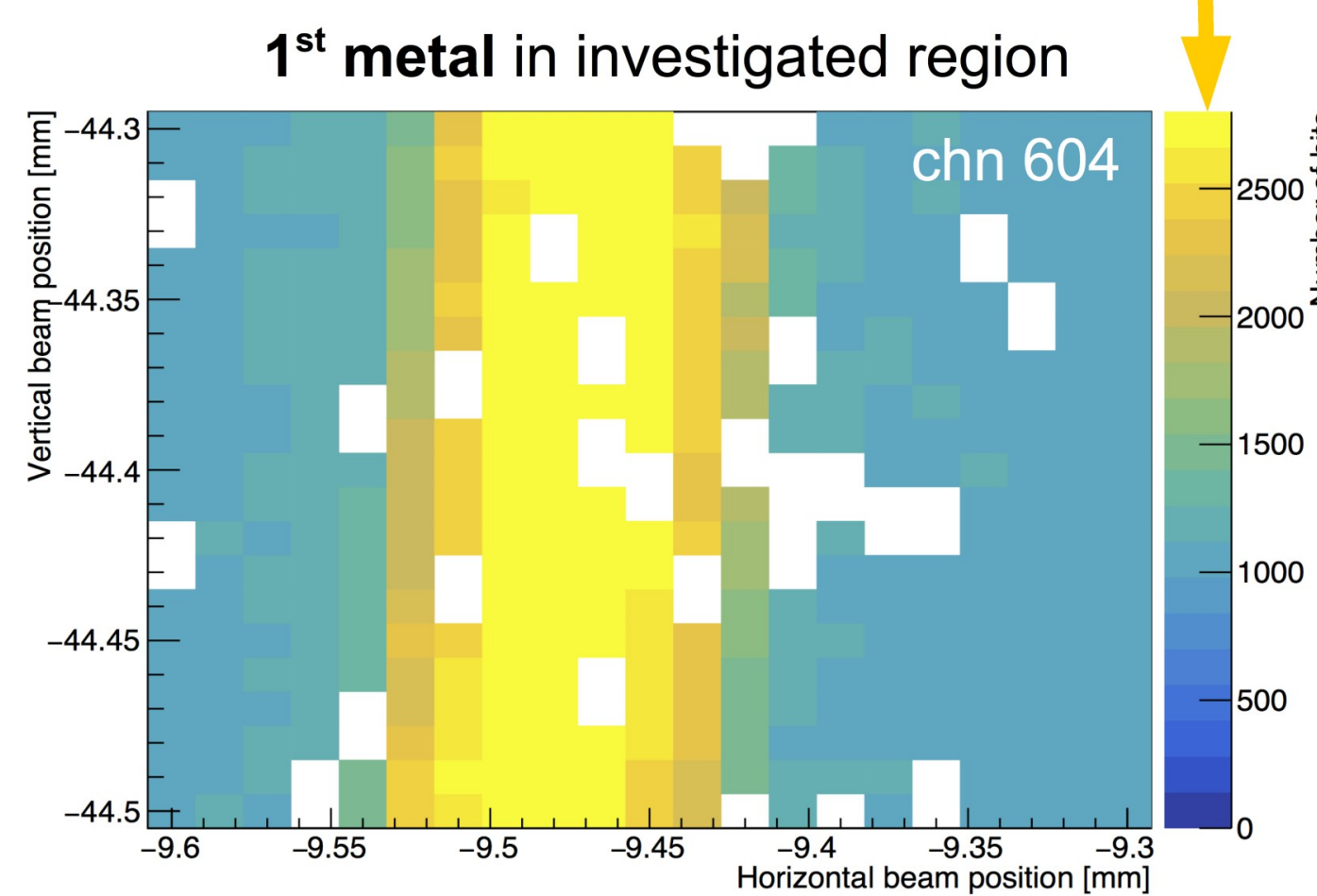

\footnotetext{
Conclusions and Outlook

- First testbeam results on sensors with embedded pitch adapters

- $15 \mathrm{keV}$ micro-focussed photon beam $\rightarrow$ no MIPs, no quantification, but good mapping out of effect

- Pick-up present in unirradiated sensors for

- low thresholds of binary readout

- EPA architecture with largest $2^{\text {nd }}$ metal area ("worst case scenario")

- Effect decreases fast towards operating threshold

- Effect possible only in EPA region (<10\% of sensor area)

- strongest in peripheral strip area

Up next:

- Compare with irradiated sensor

- Extend measurement to higher thresholds

- Repeat measurement for more favored EPA architecture

- Compare with measurements from particle testbeam
} 